\title{
The LUCAS program: detecting vegetation and traces of life in the Earthshine
}

\author{
Danielle Briot ${ }^{1}$, Luc Arnold ${ }^{2}$, Stéphane Jacquemoud ${ }^{3}$, \\ Jean Schneider ${ }^{4}$, Karim Agabi ${ }^{5}$, Eric Aristidi ${ }^{5}$, Jérôme Berthier ${ }^{4}$, \\ Erick Bondoux ${ }^{6}$, Zalpha Challita ${ }^{6}$, Denis Petermann ${ }^{6}$, \\ Cyprien Pouzenc ${ }^{6}$ and Patrick Rocher ${ }^{4}$ \\ ${ }^{1}$ Observatoire de Paris-Meudon, 61 avenue de l'Observatoire, 75014 Paris \\ email: danielle.briot@obspm.fr \\ ${ }^{2}$ Observatoire de Haute-Provence, France \\ ${ }^{3}$ Université Paris-Diderot, IPGP, France \\ ${ }^{4}$ Observatoire de Paris-Meudon \\ ${ }^{5}$ Université Nice Sophia-Antipolis \\ ${ }^{6}$ Concordia Station, Dome C, Antarctica
}

\begin{abstract}
The aim of the LUCAS program is to observe chlorophyll and atmospheric molecules in the Earthshine spectrum in order to prepare the detection of life in terrestrial extrasolar planets to be discovered. Actually, observations from Antarctica offer a unique possibility to study the variations of Earthshine spectrum during Earth rotation while various parts of Earth are facing the Moon. Special instrumentation for the LUCAS program was designed and put in the Concordia station in the Dome C. Observations are in progress.
\end{abstract}

Keywords. Astrobiology, Earth, Moon

\section{Introduction}

The detection of extrasolar planets has given a new impulse to the research of life in the Universe. In order to prepare the detection of life on Exo-Earths or SuperEarths located in the hability zone, when their images could be seen, we study the detection of terrestrial life, Earth being seen as a dot. Earthshine, which is located in the dark part of the Moon inside the crescent, corresponds to the Earth light backscattered by the non-sunlit Moon. Due to the lunar surface roughness, any place of the Earthshine reflects all the enlighted part of the Earth facing the Moon. So a spectrum of the Moon Earthshine directly gives the disk-averaged spectrum of the Earth, as could be seen extrasolar planets. In this spectrum we can see molecules in the planet atmosphere, like oxygen and ozone, which may be biologic markers, and also the chlorophyll spectrum due to the vegetation reflectivity. The chlorophyll spectrum presents a very typical sharp edge in the near-infrared, near $700 \mathrm{~nm}$, the so-called Vegetation Red Edge (VRE). Since 2002, (see Arnold et al. (2002) and Woolf et al. (2002)), several studies detected the VRE from Earthshine observations (see a review of the results in Arnold 2008). Although the values of VRE are only a few percent, we found larger values when continents are facing the Moon and smaller values in case of an ocean (see Arnold et al. (2002) and Hamdani et al. (2006)). To detect the variation of the Earthshine as a function of the Earth landscapes facing the Moon, long observational times are necessary, so as to observe the rotating Earth. This is possible only in the case of observations from very high latitude places, and even more so when near the pole, during a total diurnal cycle (nycthemere). 
Other information about Earthshine can be found in the same volume (Briot, 2009).

\section{Dedicated Instrumentation and the State of the Art}

Concordia station at the Dome $\mathrm{C}$ offers such a possibility. After checking the darkness of the sky during Earthshine observations, the LUCAS (LUmière Cendrée en Antarctique par Spectroscopie) experiment was then imagined in 2006, installed in 2007, and the first observations planned for the southern winter of 2008. A dedicated instrumentation for Earthshine spectroscopic observations was designed and built at Haute Provence and Paris-Meudon observatories. It is made off a $20-\mathrm{cm}$ diameter Schmidt-Cassegrain telescope that feeds a low resolution slit spectrograph. The spectrograph, based on a 300 grooves/mm reflecting grating, has a 500 to $900 \mathrm{~nm}$ spectral range and a resolution of about 100 at $700 \mathrm{~nm}$. The camera is a KAF402ME-based CCD detector. Tests carried out at the Haute-Provence observatory validated the instrumentation: overall optical alignment focus, and data acquisition. Due to the extreme weather conditions in Antarctica, the full instrument (telescope, spectrograph and detector) is insulated to withstand the very low temperatures that prevail in the Concordia Station. The internal temperature of the instrument is regulated thanks to a set of PT100 temperature detectors and a heater that prevent the camera shutter to cool down to negative temperatures. During the 2008 campaign, we had some problems of shutter and heat insolation. The feedback we got from the first observing campaign in 2008 was very important to detect, analyze and correct instrumental problems due to extreme temperature and extreme physical conditions. Some important instrumental improvements were carried out for the 2009 winterover campaign: new thermalization, new impermeable box, high quality (military) connections, new dome, etc. These improvements, as well as the ingenuity of the winterover observers, were very efficient and we have obtained Moon spectra, Earthshine and enlightened Moon, during each observational sequence since 2009 June solstice, that is to say during each Moon cycle since the first or second day after the New Moon, up to the First Quarter. Continuous observations times spend up to 8 hours running. Obviously, so long an observational time of the Earthshine is impossible at low or moderate latitudes.

\section{Conclusion}

Actually, LUCAS is the first program with spectroscopic observations at Dome C. We obtain Earth vegetation spectra during several hours (up to 8 hours) and we will detect variations during Earth rotation, as it will be possible in the future for extrasolar planets. As such, it is also a test for the design and improvement of small instrumentation, data collecting and management of observations in Concordia's extremely cold environnement.

\section{References}

Arnold, L. 2008, Space Sci. Revs 135, 323

Arnold, L., Gillet, S., Lardière, O., Riaud, P., \& Schneider, J. 2002, A\&\&A 392, 231

Briot, D. 2009, Highlights of Astronomy vol. 15, (this volume), Special Session 6

Hamdani, S., Arnold, L., Foellmi, C., Berthier, J., Billeres, M., Briot, D., François, P., Riaud, P., \& Schneider, J. 2006, A\&\&A 460, 617

Woolf, N. J., Smith, P. S., Traub, W. A., \& Jucks, K. W. 2002, ApJ 99, 225 